ENSINO E PESQUISA ADMINISTRAÇÃO 


\title{
AMBIENTES LABORATORIAIS PARA PESQUISAS COM JOGOS DE EMPRESAS
}

\author{
LABORATORY ENVIRONMENT WITH BUSINESS GAMES IN BRAZIL
}

Adriano Maniçoba da Silva

Instituto Federal de São Paulo

Antonio Carlos Aidar Sauaia

Universidade de São Paulo

\author{
Data de submissão: | | jan. 20 |6. Data de aprovação: \\ I 5 set. 2016. Sistema de avaliação: Double blind review. \\ Universidade FUMEC / FACE. Prof. Dr. Henrique Cordeiro \\ Martins. Prof. Dr. Cid Gonçalves Filho. Prof. Dr. Luiz Claudio \\ Vieira de Oliveira
}

\section{RESUMO}

A vantagem da utilização de ambientes laboratoriais para a realização de pesquisas tem sido evidenciada em programas de pesquisa recentes, tais como as finanças comportamentais, a economia comportamental e a economia experimental, na medida em que permitem explorar aspectos subjetivos individuais e coletivos. $O$ objetivo deste estudo foi verificar se os jogos de empresas são ambientes laboratoriais adequados para a realização de pesquisas econômicas. Foram discutidos os fundamentos teóricos da economia experimental e de jogos de empresas, de modo a identificar em que condições os jogos de empresas seriam adequados para a condução de pesquisas. Pela análise de três estudos teórico-empíricos que investigaram problemas econômicos com jogos de empresas, identificou-se que os jogos de empresas são adequados para pesquisas quando se estudam hipóteses delineadas a partir de estudos anteriores, para a realização de estudos experimentais e não experimentais, quando da utilização da instituição do leilão pós-oferta e quando o contexto da pesquisa for o organizacional. $O$ estudo permitiu reforçar as vantagens dos jogos de empresas para utilização em pesquisas.

\section{PALAVRAS-CHAVE}

Ambiente Laboratorial. Jogos de Empresas. Economia Experimental. Pesquisas econômicas. Pesquisas em Administração. 


\section{ABSTRACT}

The advantage of using laboratory environments for research has been highlighted in recent research programs such as behavioral finance, behavioral economics and experimental economics, as they explore individual and collective aspects. The aim of this study was to analyze the business games as laboratory environments for research. The theoretical foundations of experimental economics and business games were discussed in order to identify the conditions under which business games would be appropriate for research. By analysis of three empirical studies that investigated economic issues with business games we concluded that business games are suitable for research when investigating hyphotesis outlined from previous studies, to perform experimental studies and non-experimental ones, when using postted-offer auction and when the object of research is the organizational context. The study has reinforced the advantages of business games for research.

\section{KEYWORDS}

Laboratorial Environment. Business Games. Experimental Economics. Economic Research. Research in Management.

\section{INTRODUÇÃO}

Eventos recentes têm chamado a atenção para novas perspectivas práticas que devem ser incorporadas em formulações teóricas. Dentre esses eventos, pode-se citar a crise do subprime de 2008. Segundo Moore e Swift (2010), o excesso de confiança pode ter sido um de seus fatores causadores. Das formulações que tratam da inclusão de aspectos cognitivos em modelos teóricos, podem-se citar a economia comportamental, as finanças comportamentais e a economia experimental (FERREIRA, 2008). Em economia experimental, os experimentos são desenvolvidos com interesse de gerar dados em um ambiente controlado e ainda verificar se comportamentos individuais convergem com modelos econômicos teóricos (CROSON; GACHTER, 20I0, p. 124).
A utilização de jogos de empresas como ambientes laboratoriais teve aplicação inicial a partir da década de 1950, nos EUA. Apesar dos simuladores organizacionais serem modelados segundo a lógica econômica, sua utilização por mais de 50 anos para pesquisas em Administração e Economia é modesta, tendo em grande parte seu uso para treinamento e educação gerencial (FARIA et al., 2009).

Este predomínio pode ser explicado pelas recorrentes críticas relacionadas ao seu ambiente laboratorial, por comprometer a validade interna (STAINTON et al., 2010 ), ou seja, à capacidade de o ambiente representar relações de causa e efeito de maneira adequada. Apesar dessa limitação, justamente pela interação de múltiplas variáveis presentes em seu ambiente laboratorial, os jogos de empresas têm sido 
citados como verossímeis em relação ao contexto organizacional, o que aumenta a capacidade de generalização (validade externa) de resultados obtidos em pesquisas (MICHALISIIN et al., 2004). Esse fato consiste, portanto, numa vantagem do ambiente laboratorial dos jogos de empresas para pesquisas.

Diferentemente dos jogos de empresas, os ambientes laboratoriais utilizados em economia experimental têm tido maior utilização em pesquisas do que em atividades ligadas ao ensino, e maior aceitação perante a comunidade científica econômica. Esse fato pode ser evidenciado pelas premiações do Nobel, de 2002, a Vernon Smith, e, de 2012, a Alvin Roth. Apesar do uso disseminado para a produção de pesquisas econômicas, os estudos conduzidos em economia experimental são criticados por conterem limitações de validade externa, ou seja, limitações quanto à generalização dos resultados da pesquisa.

Reconhecendo os potenciais de complementaridade de cada programa de pesquisa, pode ser relevante um estudo que promova a aproximação de economia experimental e jogos de empresas. Pode-se, sobretudo, contribuir para a verificação da validade interna dos jogos de empresas e da validade externa de pesquisas econômicas. Desse modo, esse estudo teve como objetivo geral investigar se os jogos de empresas podem ser ambientes laboratoriais adequados para pesquisas econômicas.

A seção seguinte apresenta a revisão da literatura de economia experimental e jogos de empresas. Na próxima, os procedimentos metodológicos serão delineados para, então, na seção subsequente, apresentar os resultados e discussão. Por fim, conclui-se apontando limitações e futuros estudos.

\section{REVISÃO DA LITERATURA}

Sendo a principal temática deste estudo, definiu-se ambiente laboratorial como o conjunto de agentes (indivíduos) e recursos (produtos) que podem interagir em caráter experimental (SMITH, I 98I, p. 924). Também segundo este mesmo autor, define-se instituição como o mecanismo que rege a interação num mercado.A próxima seção trata da literatura de jogos de empresas.

\section{Jogos de empresas}

Jogo de empresas foi definido como ambiente laboratorial, que representa um mercado no qual empresas podem atuar, produzindo e comercializando um produto. Atrelados à definição apresentada, podem-se atribuir dois componentes importantes presentes nos jogos de empresas: (I) o simulador, que consiste no artefato, manual ou eletrônico, contendo as regras da interação, ou instituição, como definido na seção anterior; e (2) ० jogo em si, que envolve a tomada de decisão sob incerteza, que ocorre quando da interação de participantes ou de participante com a instituição (SAUAIA, 20I3). Essa definição está consonante as formulações recentes, tal como a de Greco et al. (2013, p. 647), de que participantes gerenciam de maneira virtual um empreendimento comercial ou não comercial.

Os jogos de empresas foram desenvolvidos para que proporcionassem aos participantes experiência vivencial verossímil em relação ao contexto organizacional. Cameron e Whetten (198I) ressaltam que jogos de empresas permitem que se desenvolvam estágios similares aos experimentados em organizações reais, cuja variação afeta a interpretação dos membros, de modo sistemático, 
ao longo dos períodos. O jogo de empresas tem a finalidade de representar um ambiente competitivo onde empresas laboratoriais atuam para obter resultado superior.

Também permitem um engajamento maior de indivíduos, por envolverem os participantes numa conjuntura organizacional específica, proporcionada pelo caso empresarial (SAUAIA, 20I3). O caso empresarial oferece, aos indivíduos, imersão num setor e empresa específicos, caracterizados por meio de balanços patrimoniais, demonstrativos de caixa, demonstrativos de resultados, relatórios anuais e planos de gestão. Diferentes empresas e também diferentes setores econômicos podem ser caracterizados tanto pelos parâmetros do simulador, quanto pelos materiais de divulgação e manuais desse artefato. A conjuntura organizacional, descrita no caso empresarial, proporciona envolvimento maior do participante. Para Lant (I 989), o jogo de empresas permite aumentar o interesse, o envolvimento e o entusiasmo que podem estar presentes no comportamento em organizações reais.

A possibilidade de se observar o comportamento social e obter envolvimento pessoal faz com que os jogos de empresas proporcionem medidas melhores do comportamento individual do que outras formas de coleta de dados, como o levantamento (Survey). Pelo fato de a atividade dos jogos de empresas envolver a tomada de decisões, estas podem ser coletadas e servir de insumo para pesquisas propiciando a coleta de maneira direta, não necessariamente na forma de percepção (KEYS; WOLFE, 1990, p. 323). Com os dados coletados dessa forma, é possível conduzir pesquisas no ambiente laboratorial dos jogos de empresas, cegas aos participantes.
Para que possa representar a realidade organizacional, o ambiente laboratorial dos jogos de empresas envolve a interação de múltiplas variáveis. Os simuladores que subsidiam a utilização dos jogos de empresas têm essa característica para prover maior realismo aos participantes. Como exemplo, pode-se citar o simulador SIMCO (2009), que tem mais de 30 variáveis de decisão por período. Por esse fato, o realismo que jogos de empresas proveem tem sido ressaltado como uma das suas principais características.

Apesar de a interação de múltiplas variáveis propiciar maior realismo ao destacar diversos elementos da conjuntura organizacional, a principal crítica aos jogos de empresas para utilização em pesquisas é a limitação quanto à validade interna ocasionada por esta interação (STAINTON et al., 2010).A despeito das críticas, Wolfe e Castrogiovanni (2006, p. 3I) afirmam que jogos de empresas são adequados para prover laboratórios de pesquisa para estudos controlados em estratégia.

As primeiras referências aos jogos de empresa como laboratórios de pesquisa remontam a Bruns (1962, p. 148) e Dill e Doppelt (1963). Na própria década em que os jogos de empresas tiveram utilização na área de negócios, já foram utilizados para pesquisas. Estudos pioneiros estavam interessados na relação entre a performance da empresa laboratorial e variáveis psicodemográficas. Estudos subsequentes tiveram como objeto de pesquisa problemas em Administração.

A partir de diversos estudos revisados, Halpin (2013) verificou que, no âmbito da realização de pesquisas, os jogos de empresas têm sido utilizados para estudar problemas em Administração, principalmente 
no que concerne ao processo de tomada de decisão e características dos indivíduos ou equipes, e ao seu efeito em variáveis da empresa laboratorial. A autora constatou ainda que as pesquisas realizadas neste ambiente são frequentemente de natureza correlacional. Com a finalidade de resumir os fundamentos dos jogos de empresas identificados nesta seção, a Figura I apresenta $\circ$ processo do funcionamento dos jogos de empresas.

Nos jogos de empresas, equipes são formadas e tomam decisões acerca de uma empresa laboratorial, que está inserida numa conjuntura organizacional pelo caso empresarial. O simulador organizacional, caracterizado pela interação de múltiplas variáveis, mescla as decisões e emite relatórios de desempenho que retornam às equipes para que haja a avaliação do desempenho. Novamente, as equipes incorrem em outro período decisório, seguido por nova análise de resultados. Esse ciclo se repete até que chegue ao fim a quantidade de pe- ríodos determinados. Cabe apontar também que, para assegurar a eficácia educacional na condução dos jogos de empresas, são necessárias atividades concernentes ao ministrante e ao simulador envolvidos na interação. A próxima seção apresentará os fundamentos do programa de pesquisa da economia experimental.

\section{Economia experimental}

O primeiro estudo sobre equilíbrio de mercado, conduzido em ambiente laboratorial, de que se tem notícia em Economia, é atribuído a Chamberlin (1948). Como indicava a teoria, esperava-se que a média de preços e quantidades negociados atingisse o nível indicado pela intersecção das curvas de oferta e demanda. Porém, esse fato não ocorreu, frustrando o autor, que desistiu dos ambientes laboratoriais. Alguns anos mais tarde, um dos estudantes de Chamberlin, Vernon Smith, intrigado com o fracasso do experimento, conduziu novos estudos laboratoriais em que os resultados convergi-

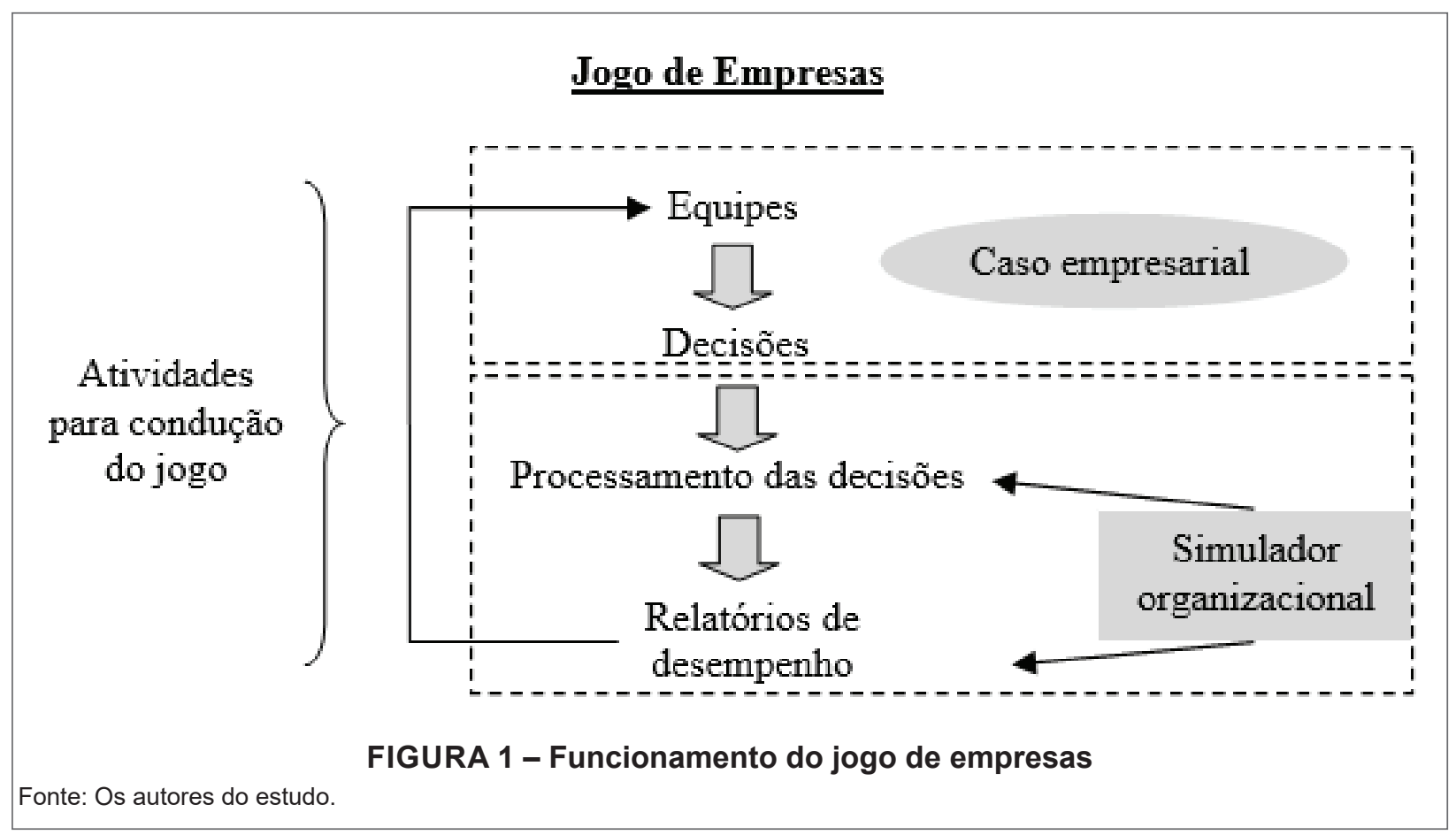


ram com níveis teóricos previstos (SMITH, 1962). Smith centralizou a informação das negociações, de modo que os preços transacionados fossem informação pública. Esse formato institucional, para verificar o equilíbrio de mercado em laboratório, ficou conhecido como leilão duplo "double auction" (DAVIS; HOLT, 1992, p. 7). A convergência do resultado obtido por Smith contribuiu para o reconhecimento da importância do método laboratorial em Economia, culminando com o prêmio Nobel em 2002.

Para avançar no reconhecimento do método laboratorial, houve considerável cuidado com a formalização dos experimentos econômicos. Contribuindo para formalizar o ambiente laboratorial para pesquisa econômica, Smith (1982, p. 924) faz uma analogia de um experimento com um sistema microeconômico e destaca que esse sistema tem dois componentes distintos: ambiente e instituição.

$O$ ambiente envolve um conjunto de agentes $\{I, \ldots, N\}$, que dispõem de uma lista de $K+I$ recursos (commodities) $\{0, I, \ldots, K\}$ que por sua vez possuem atributos $\omega^{i}$. As características de cada agente podem ser representadas com uma função utilidade $u^{i}$, e os agentes possuem ainda tecnologia que pode ser considerada como conhecimento ou habilidades $T$. Desse modo, o ith agente é caracterizado pelo vetor $\mathbf{e}^{i=}$ $\left(\mathbf{u}^{i}, \mathbf{T}^{i}, \boldsymbol{\omega}^{i}\right)$. Resumindo, o ambiente microeconômico é definido pela coleção de características, $\mathrm{e}=\left(\mathrm{e}^{1}, \ldots, \mathrm{e}^{\mathrm{N}}\right)$, dos agentes.

Instituição consiste num conjunto de regras e mecanismos específicos que governam um ambiente laboratorial (SMITH, 1982; DAVIS; HOLT, 1992). A instituição contém as regras da propriedade privada nas quais os agentes podem se comunicar, realizar trocas ou transformar os recursos de acordo com sua utilidade. Para o autor, a instituição envolve: (I) uma linguagem $M=\left(M^{1}, \ldots . . M^{N}\right)$ que é composta de mensagens $\mathrm{m}=\left(\mathrm{m}^{1}, \ldots, \mathrm{m}^{\mathrm{N}}\right)$, onde $\mathrm{m}^{\mathrm{i}}$ é um elemento de $M$, que podem ser enviadas pelo agente i. Uma mensagem $(\mathrm{m})$ pode ser um lance, uma oferta ou um aceite; (2) um conjunto $\mathrm{H}=\left(\mathrm{h}^{1}(\mathrm{~m}), \ldots, \mathrm{h}^{\mathrm{N}}(\mathrm{m})\right)$ de regras de alocação para cada agente i. A regra $h^{i}(m)$ determina a alocação final dos recursos para cada $i$ como função das mensagens enviadas por todos os agentes. Desse modo, pode haver uma troca de mensagens as quais precedem a alocação; (3) um conjunto $C=\left(c^{\prime}(m), \ldots, c^{N}(m)\right)$ de regras de imputação de custo. A regra $c^{i}(m)$ determina o pagamento que deverá ser feito por cada agente em unidades monetárias como função das mensagens enviadas por todos os agentes; e (4) $O$ conjunto $G=\left(g^{\prime}\left(t_{0}, t,-\right.\right.$ $\left.T), \ldots, g^{N}\left(t_{0}, t, T\right)\right)$ de regras de ajustamento do processo. No geral, essas regras de ajustamento consistem na regra de início $g^{i}\left(t_{0}, . .,\right)$, que especifica $\circ$ tempo ou as condições sob as quais a troca de mensagens deve se iniciar, uma regra de transição gi(., t..), que governa a sequência da troca de mensagens, e uma regra de parada gi(..,. $T$ ) sob a qual a troca de mensagens é finalizada (e as alocações se iniciam).

Smith (1982, p. 93I) afirmou ainda que a condição necessária para um experimento microeconômico é o controle das preferências. Este controle deve ser baseado numa estrutura de incentivo que deve estar contida no sistema de direito de propriedade para induzir a um comportamento baseado numa estrutura monetária. Smith salienta ainda quatro princípios fundamentais para que haja controle das preferências que são o princípio da não saciedade, da saliência, da dominância e da privacidade. 
Friedman e Sunder (1994) consideram que esses princípios constituem a teoria do valor induzido, ou seja, a forma pela qual um experimento econômico controla as preferências dos participantes. O princípio de não saciedade pode ser exemplificado pela seguinte situação: numa escolha entre duas alternativas: se a primeira oferece retorno médio maior que a segunda, então a primeira será sempre escolhida. Em outras palavras, o indivíduo não é saciável em relação ao retorno do experimento. Saliência se refere ao claro entendimento do retorno que o participante deve ter como resultado de suas decisões. Dominância consiste no fato de a estrutura de recompensa do experimento compensar os custos subjetivos associados à participação do sujeito em suas atividades. Privacidade se refere ao fato de cada jogador receber informação apenas sobre suas próprias alternativas de decisão e recompensa. A Figura 2 resume os principais elementos que compõem um experimento econômico.
Um experimento econômico (FIGURA 2) contém um sistema microeconômico e proporciona o controle das preferências dos participantes. Os agentes representados possuem utilidades e tecnologia (conhecimento), e interagem conforme as regras de ajustamento, que dispõem sobre recursos iniciais e início/fim da atividade. A seguir, os agentes emitem suas respectivas mensagens (linguagem), conforme as regras de alocação que designam os recursos e geram os custos decorrentes das mensagens atribuídos pelas regras de imputação de custo.

Para que o controle de preferências seja satisfatório, a compensação advinda das mensagens, após a aplicação das regras de imputação de custo, deve variar a preferência do agente de acordo com o resultado esperado, e o indivíduo sempre preferirá maior retorno médio (não saciedade). $O$ aumento da preferência do agente também deve ser maior que quaisquer outros resultados positivos ou negativos advindos de

\section{Controle de Preferências $\quad \underline{\text { Sistema Microeconômico }}$}

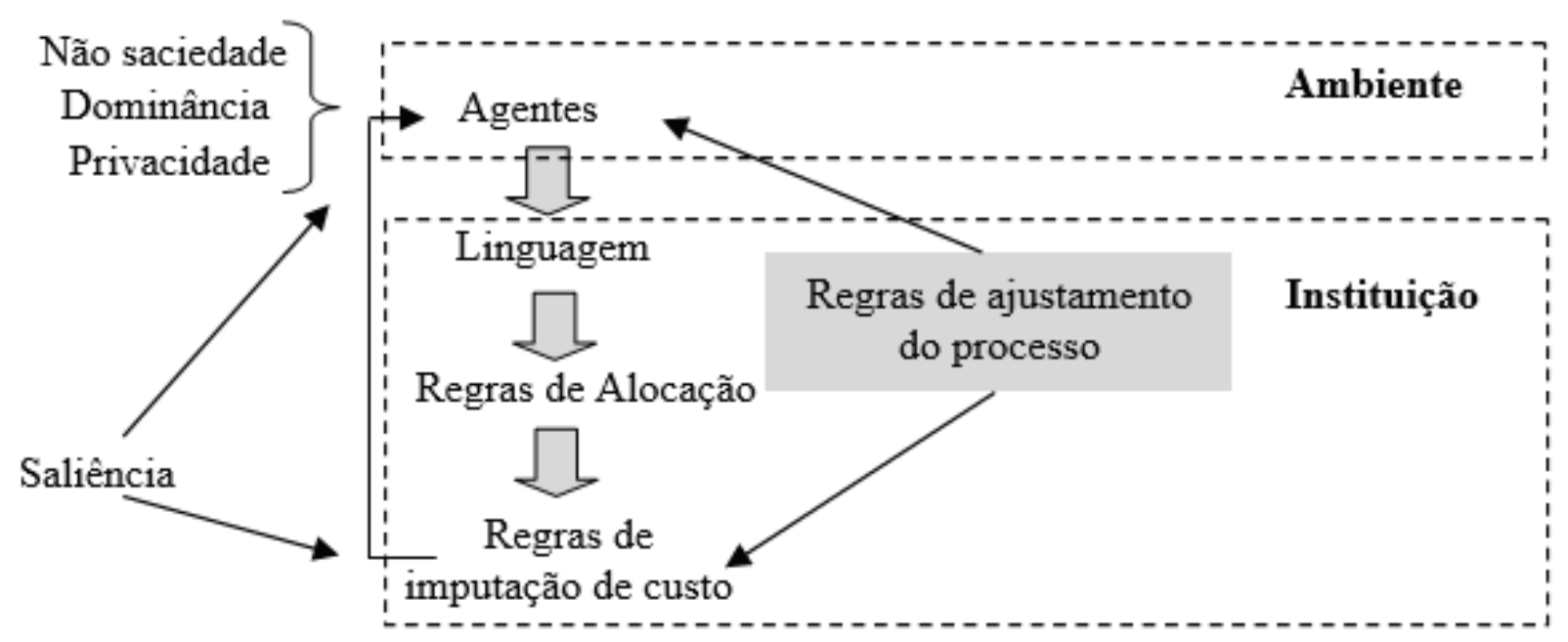

FIGURA 2 - Funcionamento de um experimento econômico

Fonte: Os autores do estudo. 
outro estímulo (dominância). Ainda, cada participante deve conhecer apenas suas alternativas de recompensa advindas de suas mensagens (privacidade). Por fim, os agentes devem entender os resultados das regras de imputação de custo provenientes das regras de alocação e respectivamente das mensagens (saliência).

Os experimentos econômicos podem ainda variar num continuum representado pelos extremos: experimento de laboratório e experimento de campo natural. Essa taxonomia foi proposta por Harrison e List (2004), da sintetização de diversos estudos anteriores (QUADRO I).

Segundo Harrison e List (2004), o experimento de laboratório tem como sujeito participante o estudante recrutado ou proveniente de turmas do próprio pesquisador. O cenário do experimento é abstrato, ou seja, os participantes se imaginam numa situação e utilizam recursos fictícios. As regras que governam o experimento advêm da instituição utilizada e são impostas aos participantes. Os mesmos sabem que participam de um experimento e realizam tarefas específicas solicitadas. As demais categorias são variações do experimento de laboratório e se caracterizam pela inclusão de elementos no desenho deste tipo de experimento, de modo que tenham maior validade externa, ou seja, possam representar a realidade de maneira mais verossímil em relação aos ambientes estritamente laboratoriais. A próxima seção discute os principais fundamentos abordados dos programas de pesquisa de jogos de empresas e economia experimental.

\section{Síntese da revisão da literatura}

Ao comparar o funcionamento dos jogos de empresas com os ambientes utilizados em economia experimental, pode-se destacar alguns apontamentos. Quanto ao tipo de instituição utilizada, identificou-se, a partir de uma relação de quinze tipos diferentes elencados por Davis e Holt (1992), que os jogos de empresas se enquadram numa instituição específica denominada Leilão pós-oferta. Cada equipe representa uma empresa que geralmente compete com outras concorrentes, oligopólio, ou atua de maneira isolada num monopólio. A quantidade vendida é modelada pelo simulador por meio de uma curva de demanda. As empresas tomam decisões simultâneas; o ministrante opera o simulador e executa o período vigente. Num primeiro momen-

QUADRO 1 - Classificação dos experimentos econômicos

\begin{tabular}{|c|c|c|c|c|}
\hline Elementos & $\begin{array}{c}\text { Experimentos de } \\
\text { laboratório }\end{array}$ & $\begin{array}{c}\text { Experimento de } \\
\text { campo artefactual }\end{array}$ & $\begin{array}{c}\text { Experimento de } \\
\text { campo framed }\end{array}$ & $\begin{array}{c}\text { Experimento de } \\
\text { campo natural }\end{array}$ \\
\hline Sujeito & Estudante & Profissional & Profissional & Profissional \\
\hline Cenário & Abstrato & Abstrato & Real & De campo \\
\hline Regras & Impostas & Impostas & De campo & Não \\
\hline $\begin{array}{c}\text { Conhecimento do experimento } \\
\text { Tarefas específicas ao } \\
\text { experimento }\end{array}$ & Sim & Sim & Sim & Não \\
\hline Tipo de validade & Interna & Sim & & Externa \\
\hline
\end{tabular}

Fonte: adaptado de Harrison e List (2004) 
to, vendedores decidem, dentre outras variáveis, o preço, de maneira simultânea, e, em seguida, compradores modelados exercem seu papel sob a regra "leve ou deixe".

Também a partir da literatura revisada na seção “Jogos de empresas”, em vista dos argumentos apresentados, conclui-se que, enquadrados como leilão pós-oferta, os jogos de empresas poderão figurar como ambiente laboratorial, para que estudos que explorem esta instituição possam se beneficiar da possibilidade de obterem maior validade externa, num ambiente laboratorial com interação de múltiplas variáveis, onde indivíduos possam exibir seu comportamento social, e envolvimento pessoal com o caso empresarial. Neste estudo, afirma-se que essas três características destacadas, ao possibilitarem maior validade externa, permitem representar, em certa medida, o contexto organizacional, e são condições para a realização de pesquisas com jogos de empresas.

Ainda segundo Plott e Smith (2008, p. 5), o leilão pós-oferta foi uma das instituições mais utilizadas em estudos pioneiros de economia experimental. Para os autores, é o modelo mais indicado para estudos de conduta em oligopólio e poder de mercado, pois possui a propriedade de convergência de preços supra-competitivos mais eficaz em relação ao leilão-duplo. Desse modo, o ambiente laboratorial dos jogos de empresas será propício para estudos de oligopólio que investiguem questões relacionadas ao poder de mercado. A próxima seção apresenta os procedimentos metodológicos adotados para a verificação dessas condições nos estudos revisados.

\section{METODOLOGIA}

Para atingir o objetivo do estudo, utili- zou-se uma abordagem qualitativa de pesquisa que, sob a análise de estudos empíricos anteriores, buscou indícios e evidências da adequação do ambiente laboratorial dos jogos de empresas para a realização de pesquisas nas condições identificadas na seção anterior. Quanto ao método empregado, destaca-se o bibliográfico (GONÇALVES, 2007), pois consistiu na análise de estudos precedentes.

Foi realizado um levantamento dos artigos de jogos de empresas nas publicações no Brasil e no exterior, ao longo do período de 2010 a 2014.A pesquisa bibliográfica ocorreu em julho de 2015 e consistiu na busca de artigos em bases de dados nacionais e internacionais. $O$ processo de busca e escolha dos artigos foi dividido em três etapas. A etapa inicial consistiu na busca de estudos com as palavras-chave determinadas. $\mathrm{Na}$ segunda etapa, os resumos dos artigos foram lidos com a finalidade de identificar pesquisas conduzidas com temas de Economia. Por fim, na terceira etapa, foram escolhidos estudos que atendiam às condições identificadas na seção "Síntese da revisão da literatura", ou seja, estudos que tiveram como objeto de pesquisa pelo menos um dos seguintes itens: a interação de múltiplas variáveis; comportamento social ou envolvimento pessoal dos sujeitos participantes; análise do contexto organização; análise de questões relativas ao oligopólio e poder de mercado.

A busca de artigos publicados no exterior foi realizada a partir da palavra-chave "business games". De pesquisa realizada na base de dados Ebsco, tal como fez Silva (20I5), resultaram 29 artigos, sendo que dois abordaram temas de economia. Entretanto, conforme se verificou na terceira etapa, nenhum deles atendeu às condições 
elencadas no parágrafo anterior. Com procedimento similar ao de Ribeiro, Ferreira e Costa (20I4), também foi feita pesquisa na base de dados ISI Web of Science. A busca resultou em 43 artigos, sendo que nenhum tratou de temas de Economia.

Para que a pesquisa no âmbito nacional tivesse representatividade, foi realizada busca nos artigos publicados nos anais dos eventos Enanpad e Semead, e também em periódicos na base de dados Spell (Scientific Periodicals Electronic Library). A escolha dos eventos se deu por sua representatividade na área de Administração, tal como feito no estudo de Battistella, Schuster e Dias (20/2). A base de dados Spell tem incorporado importantes periódicos, tal como apontaram Schmitt et al. (20I3) e tal como utilizado em Peçanha e Lizuka (20 I4).Tratase de uma base de dados que disponibiliza artigos dos principais periódicos nacionais.

A busca nas bases de dados do Enanpad, do Semead e do Spell, utilizando a palavra-chave jogos de empresas, no intervalo de tempo de 2010 a 2014, resultou em 34 artigos, sendo 14 da base de dados Spell, cinco (5) dos Anais do Enanpad e 15 dos Anais do Semead. Na verificação dos temas de economia, segunda etapa, restaram quatro (4) artigos, sendo que três (3) atenderam às condições da terceira etapa. Foram escolhidas as pesquisas de Silva e Sauaia (20I2), de Silva e Adriano (20I4), do Semead, e de Silva e Sauaia (20/3), da base de dados Spell. O estudo de Sauaia (20II), no Enanpad, foi descartado por não atender às condições da terceira etapa. $A$ justificativa da escolha dos estudos é exibida no Quadro 2.

No estudo de Silva (20I4), doravante denominado de Estudo I, elaborado sob - enfoque da abordagem neoclássica, foi verificado se os preços praticados em monopólio diferiram dos preços de oligopólio. Como sinaliza a teoria, os resultados convergiram para os estudos realizados a partir de Smith (198I). Já no estudo de Silva e Sauaia (20/3), que será denominado por Estudo 2, conduzido sob o programa de pesquisa da organização industrial, foram comparados sinal e magnitude do desvio do modelo de aferição de poder de mercado da Nova Organização Industrial Empírica (NOIE), em relação à Estrutura-Conduta-Desempenho (ECD), com base em dados contábeis. O resultado foi condizen-

QUADRO 2 - Justificativa da escolha dos estudos analisados

\begin{tabular}{|l|l|c|}
\hline Análise das Condições & Justificativa & Estudo(s) \\
\hline $\begin{array}{l}\text { Conduzir experimentos econômicos consagrados no } \\
\text { ambiente laboratorial dos jogos de empresas }\end{array}$ & $\begin{array}{l}\text { Com a finalidade de verificar se o ambiente } \\
\text { laboratorial dos jogos de empresas permite replicar } \\
\text { resultados anteriores, validade interna, conforme } \\
\text { realizado por Horton et al. (2011) representando o } \\
\text { leilão pós-oferta }\end{array}$ & Silva (2014) \\
\hline $\begin{array}{l}\text { Conduzir pesquisas econômicas, não } \\
\text { experimentais no ambiente laboratorial dos jogos } \\
\text { de empresas }\end{array}$ & $\begin{array}{l}\text { Conforme realizado em pesquisas anteriores com } \\
\text { jogos de empresas }\end{array}$ & $\begin{array}{c}\text { Silva e Sauaia } \\
\text { (2012) e Silva e } \\
\text { Sauaia (2013) }\end{array}$ \\
\hline $\begin{array}{l}\text { Conduzir pesquisas econômicas sobre a temática } \\
\text { de oligopólio e poder de mercado no jogo de } \\
\text { empresas }\end{array}$ & Conforme sugerido por Plott e Smith (2008) & $\begin{array}{c}\text { Silva e Sauaia } \\
(2013)\end{array}$ \\
\hline $\begin{array}{l}\text { Conduzir pesquisa econômica analisando o efeito } \\
\text { do contexto organizacional presente no jogo de } \\
\text { empresas }\end{array}$ & $\begin{array}{l}\text { Consiste numa vantagem dos jogos de empresas } \\
\text { proporcionados pela interação de múltiplas } \\
\text { variáveis, comportamento social e envolvimento } \\
\text { com o caso empresarial }\end{array}$ & $\begin{array}{c}\text { Silva e Sauaia } \\
\text { (2012) }\end{array}$ \\
\hline
\end{tabular}

Fonte: Os autores do estudo. 
te com estudo de Clay e Troesken (2003). Por fim, em Silva e Sauaia (20I2), Estudo 3, conduzido sob o programa da economia comportamental, foi examinado se o desempenho organizacional teve relação com o nível de confiança das estimativas subjetivas de indivíduos, como predito por Moore e Healy (2008), o que foi confirmado. A próxima seção apresenta os resultados obtidos pela análise dos estudos empíricos.

\section{DISCUSSÃO}

\section{Jogo de empresas e a validade interna}

Seguindo a definição de Frechette (20I I), ao assumir que a validade interna consiste na capacidade de atribuição correta de causa e efeito no ambiente laboratorial, o Estudo I evidenciou que a diferença de preços adveio da estrutura de mercado. Da mesma forma, no Estudo 2, os testes de diferença de média realizado pela Anova apresentou indícios de que a competição diminuiu no segundo ano, como decorrência da mudança no regime político. Por fim, no Estudo 3, o nível de confiança de estimativas subjetivas de indivíduos se mostrou relacionado com o desempenho organizacional.

Além de os resultados obtidos estarem de acordo com estudos anteriores, outro ponto central pode atenuar a crítica de validade interna nos jogos de empresas. Tal ponto se refere à similaridade dos estudos conduzidos nos jogos de empresas com os experimentos de campo naturais. Tal similaridade é evidenciada principalmente pelo fato de os participantes envolvidos nos jogos de empresas não terem conhecimento de possíveis pesquisas conduzidas neste ambiente.

De acordo com Heukelom (20II), a discussão sobre o trade-off entre validade interna e validade externa adveio da Psico- logia, para ser um tópico recorrentemente abordado nos trabalhos em Economia, principalmente após o advento da economia experimental. $O$ uso do termo validade, na pesquisa experimental, foi difundido pelo professor Donald Campbell, dando início à classificação de experimentos em mais ou menos válidos. Segundo Heukelom (20II), tem havido extensa discussão sobre o fato de estudos frequentemente afirmarem que o experimento de laboratório tem maior validade interna, com validade externa comprometida, e o experimento de campo natural tem maior validade externa, com validade interna comprometida.

List (20I I) discorda de tais assertivas por defender que um desenho de pesquisa bem elaborado permite que se controlem ambas as validades no experimento de campo natural, principalmente pelo fato de os elementos de validade interna serem controlados a partir de estudos anteriores, ou por um modelo teórico satisfatório. Desse modo, autores contemporâneos seguem essa concepção ao aceitar que o experimento de campo natural seja válido, internamente, por formular hipóteses a partir de experimentos de laboratório, e, externamente, por ocorrerem no cenário real.

Para se ter uma ideia do avanço, autores contemporâneos consideram que $\circ$ experimento de campo natural seja um experimento puro, diferentemente do que ocorria na década de 2000 , em que os experimentos de campo naturais eram frequentemente denominados como quase -experimentos, em que não é possível controlar as causas que impactam uma variável de interesse (HEUKELOM, 20II). Desse modo, ao corroborar hipóteses delineadas a partir de estudos anteriores, frequentemente elaborados com desenho simpli- 
ficado, o experimento de campo natural permite que se obtenha validade externa, pressupondo validade interna. Conclui-se que os resultados obtidos no ambiente laboratorial dos jogos de empresas serão válidos externamente, em relação a esses três elementos que propiciam o contexto organizacional, pressupondo validade interna a partir de estudos anteriores.

Tal como fizeram Harrison e List (2008), ao conduzirem um estudo simplificado, seguido de um experimento de campo natural, a adoção do jogo de empresas como ambiente laboratorial de pesquisa deve preceder a condução de experimentos de laboratórios ou estudos não experimentais, com desenho simplificado, quando da análise de hipóteses derivadas de modelos estritamente teóricos. Desse modo, verifica-se que os jogos de empresas são adequados para estudos que busquem aumentar a validade externa das hipóteses analisadas, no que tange ao contexto organizacional.

\section{Jogos de empresas e o contexto organizacional}

Acredita-se que, pelo fato de utilizar ambiente laboratorial com interação de múltiplas variáveis, pela atuação em equipe permitir o comportamento social e o caso empresarial propiciar o envolvimento do indivíduo, a principal vantagem do am- biente laboratorial dos jogos de empresas talvez seja a possibilidade de se realizar pesquisas num ambiente contextualizado.

Contribuindo com a possibilidade de aumentar a validade externa, partindo da taxonomia de Harrison e List (2004) (QUADRO I), este estudo encontrou evidências e indícios de que os jogos de empresas podem ser adotados como ambientes para a condução de estudos laboratoriais, contendo características do ambiente de laboratório, tais como o perfil do sujeito, que é o estudante, e o cenário, que se configura abstrato, e também possuindo características do experimento de campo natural, devido ao fato de as regras serem de campo, de o participante não ter conhecimento de pesquisa e por não haverem tarefas específicas à pesquisa, nas atividades de decisão.

Além das características que aproximam o ambiente laboratorial do jogo de empresas dos experimentos de campo naturais, verifica-se que possui a vantagem de propiciar o contexto organizacional. Acredita-se que 0 contexto se mostrou presente nos estudos analisados, conforme pode ser visualizado no Quadro 3. Tal contexto permitiu que os resultados apresentassem vantagens se comparados aos de estudos anteriores.

Verifica-se que, nos estudos conduzidos, o contexto organizacional proporcionado pelo comportamento social, pela interação

QUADRO 3 - Resultados proporcionados pelo contexto nos três estudos empíricos

\begin{tabular}{|c|c|c|c|}
\hline Estudos & Elemento do contexto & Resultados & Evidências e indícios \\
\hline 1 & $\begin{array}{c}\text { Comportamento social; interação } \\
\text { de múltiplas variáveis }\end{array}$ & $\begin{array}{c}\text { Padrões comportamentais presentes na } \\
\text { variação intra-sujeitos; Validade externa do } \\
\text { efeito de variação na estrutura }\end{array}$ & $\begin{array}{c}\text { Não rejeição de H2 e H1 } \\
\text { respectivamente }\end{array}$ \\
\hline 2 & $\begin{array}{c}\text { Interação de múltiplas variáveis; } \\
\text { comportamento social }\end{array}$ & $\begin{array}{c}\text { Comparar o poder de mercado (NOIE e ECD) } \\
\text { da competição de H6; redução } \\
\text { ano segundo }\end{array}$ \\
\hline 3 & $\begin{array}{c}\text { Envolvimento pessoal com o } \\
\text { caso empresarial }\end{array}$ & $\begin{array}{c}\text { Expectativa identificada com 2 de } 6 \\
\text { respondentes de cada empresa }\end{array}$ & Não rejeição de H7 \\
\hline
\end{tabular}

Fonte: Os autores do estudo. 
de múltiplas variáveis e o envolvimento com o caso empresarial permitiram alguns resultados específicos, condizentes com ou contrários a estudos anteriores. No Estudo I, verificou-se a não diminuição dos preços praticados, ao variar a estrutura de monopólio para oligopólio, como preconizado por Engel (2007), evidenciando que o comportamento social foi condizente com o comportamento individual. Também foi possível corroborar o resultado dos experimentos clássicos elaborados a partir de Smith (198I), com a obtenção do efeito da estrutura de mercado nos preços praticados sob a interação de múltiplas variáveis.

No Estudo 2, a interação de múltiplas variáveis permitiu, ao propiciar a análise de deslocadores de oferta e demanda, comparar a eficácia do modelo NOIE em relação ao modelo ECD para aferir o poder de mercado.Apesar de a principal hipótese ter sido rejeitada, foi possível identificar resultado condizente com o estudo de Clay e Troesken (2003), apontando a necessidade de estudos futuros. Também foram identificados indícios da relevância do comportamento social no fato da diminuição da competição a partir do segundo ano de operações.

Por fim, no Estudo 3, verificou-se que, mesmo sob atuação social, o nível de confiança das estimativas individuais foi correlacionado com o desempenho da empresa laboratorial, permitindo inferir que esta propiciou envolvimento pessoal individual suficiente para afetar as estimativas subjetivas.

Os benefícios do contexto organizacional propiciado pelos jogos de empresas podem fazer avançar estudos anteriores com desenho simplificado (LOEWENSTEN, 1999). Há evidências, em estudos anteriores, de que as decisões que ocorrem sob contexto têm mais qualidade do que decisões tomadas em atividades descontextualizadas. Ortmann e Gigerenzer (1997) identificaram que há melhora significativa na capacidade de raciocínio, tal como acertos em teste de lógica, quando a atividade é contextualizada. Tal como ocorre em economia comportamental, estudos contextualizados podem ser utilizados para identificar possíveis alterações em resultados em relação a atividades descontextualizadas. Analisando os estudos conduzidos sob essa perspectiva, o único a rejeitar a principal hipótese foi o Estudo 2. Tal fato pode ter ocorrido em virtude do contexto. Porém, para se afirmar categoricamente que o contexto pode ter interferido nesse resultado, futuros estudos são necessários.

Também como evidenciou Zizzo (20l0), os jogos de empresas podem ser adequados para atenuar o efeito demanda do experimentador. Verificou-se que os estudos analisados tentaram mitigar esse efeito, na realização do Estudo I, quando da mensuração do nível de confiança das estimativas, pelo fato dessa atividade ter iniciado junto com as atividades do jogo de empresas.

\section{Jogos de empresas e seu sistema microeconômico}

Assim como destacado por Smith (1982), é desejável que um experimento econômico emule um sistema microeconômico.Também nos jogos de empresas, tal fato é necessário, uma vez é que o objetivo é fazer com que os participantes atuem num mercado, assumindo o papel de gestores de empresas. Partindo da formalização de Smith (1982), tal como apresentado na seção "Economia experimental", um sistema microeconômico consiste na composição do ambiente, formado pelos agentes, 
e da instituição, que contém a linguagem, as regras de alocação, as regras de imputação de custo e as regras de ajustamento do processo.

Conforme foi discutido na seção "Síntese da revisão da literatura", o simulador organizacional, juntamente com o jogo, propicia emular a instituição do leilão pós-oferta. Como os estudos utilizaram o mesmo simulador organizacional, verificou-se que este possibilitou a representação de um sistema microeconômico, onde cada elemento da instituição e do ambiente foi relacionado com os jogos de empresas conduzidos conforme Quadro 4.

Como pode ser visualizado no Quadro 4, em jogos de empresas são formadas equipes funcionais, que assumem o papel de gestores de uma empresa no mercado, representando $o$ agente. $A$ linguagem consiste no conjunto de decisões sobre que, a cada período, cada equipe deve deliberar. Com as mensagens emitidas pelas equipes, o simulador, com as regras de alocação representada pela curva de demanda em função das variáveis de decisão, aloca principalmente a quantidade a ser vendida por cada empresa e a destinação de investimentos (decisões de investimento em equipamentos, compra de matéria-prima e dividendos). A partir da quantidade vendida e da destinação dos investimentos, são atribuídos receitas e custos às empresas advindos de suas decisões, permitindo que a mesma obtenha um estado patrimonial.

Em relação a estudos anteriores, em economia experimental, no que tange à instituição, reconhece-se, nos estudos analisados, a definição de parâmetros de equilíbrio tal como evidenciada no Estudo I, que objetivou definir níveis teóricos de comparação, em que foi utilizado o preço de equilíbrio e o equilíbrio de Nash. Futuros estudos poderiam avançar nesta temática, seja na identificação de tais parâmetros, seja na parametrização do simulador, de modo que tais parâmetros sejam definidos a priori.

Outro elemento necessário a um experimento econômico, e consequentemente importante nos jogos de empresas, é o controle de preferências que será abordado na seção seguinte.

\section{QUADRO 4 - Elementos do sistema microeconômico dos jogos de empresas}

\begin{tabular}{|c|c|c|}
\hline Elementos & Em economia experimental & Nos jogos de empresas utilizados nesta pesquisa \\
\hline Ambiente & $\begin{array}{l}\text { Agentes com utilidade, habilidades e } \\
\text { recursos; } \mathrm{e}^{i}=\left(\mathrm{u}^{i}, \mathrm{~T}^{i}, \mathrm{\omega}^{i}\right)\end{array}$ & $\begin{array}{l}\text { Equipes funcionais (PRES; MKT; PLAN; FIN; PROD; RH) com } \\
\text { utilidade coletiva, habilidades individuais e recursos coletivos } \\
\text { (caso empresarial) }\end{array}$ \\
\hline \multirow{4}{*}{ Instituição } & Linguagem; $\mathrm{M}=\left(\mathrm{M}^{1}, \ldots . . \mathrm{M}^{\mathrm{N}}\right)$ & $\begin{array}{c}\text { Decisões: Preço; Marketing; P\&D; Programação da Produção; } \\
\text { Investimento em equipamentos; Manutenção; Compra de } \\
\text { matéria-prima; Dividendos; e Outros gastos }\end{array}$ \\
\hline & $\begin{array}{l}\text { Regras de alocação; } \\
H=\left(h^{1}(m), \ldots, h^{N}(m)\right)\end{array}$ & Função de demanda modelada \\
\hline & $\begin{array}{l}\text { Regras de imputação de custo; } \\
\qquad C=\left(c^{1}(m), \ldots, C^{N}(m)\right)\end{array}$ & Regras econômicas do simulador \\
\hline & $\begin{array}{l}\text { Regras de ajustamento do processo; } \\
\qquad \mathrm{G}=\left(\mathrm{g}^{1}\left(\mathrm{t}_{0}, \mathrm{t}, \mathrm{T}\right), \ldots, \mathrm{g}^{\mathrm{N}}\left(\mathrm{t}_{0}, \mathrm{t}, \mathrm{T}\right)\right)\end{array}$ & $\begin{array}{c}\text { Define o início e a quantidade de períodos em cada sessão, se } \\
\text { houver mais de uma. }\end{array}$ \\
\hline
\end{tabular}

Fonte: Os autores do estudo. 


\section{Jogos de empresas e o controle de preferências}

Nos jogos de empresas analisados, verificou-se que os princípios que asseguram o controle das preferências num experimento econômico não foram violados. Tal como pode ser verificado no Quadro 5, o princípio da não saciedade foi controlado pela utilização da TIR (Taxa Interna de Retorno) como indicador de desempenho. A saliência foi reforçada pela realização de testes objetivos em relação às variáveis do simulador, bem como na realização das rodadas-teste e preenchimento da análise anual de resultados. A dominância foi assegurada pela realização das decisões em sala de aula e a privacidade foi proporcionada pelo acesso individual aos relatórios de desempenho da empresa. $O$ princípio da saliência também foi assegurado pelas atividades na condução do jogo de empresas, conforme indicado por Lane (1995). Dessas atividades, a realização do debriefing após cada período foi fundamental para dirimir dúvidas.

A utilização da TIR como indicador de desempenho permitiu assegurar que o desempenho superior seria sempre preferível a qualquer outro retorno. É possível que, caso o crédito de disciplina tivesse sido atrelado ao desempenho com base na comparação de posições a cada período, o princípio da não saciedade teria sido violado, pois bastaria a empresa atingir a primeira posição para ser indiferente lucrar mais. A utilização da TIR como avaliação do desempenho da empresa a torna relevante na composição da nota final do jogo.A próxima seção resume as principais contribuições dos jogos de empresas identificadas nesta pesquisa.

\section{Contribuições dos jogos de empre- sas para Administração e Economia}

A partir da verificação de que os jogos de empresas atenderam aos objetivos de pesquisa nas condições especificadas, entendeu-se que os mesmos podem resultar em contribuições para Administração e Economia. No âmbito teórico em Administração, identifica-se como principal contribuição o avanço na formalização do ambiente laboratorial dos jogos de empresas, que permitiu atenuar as limitações de validade interna. Também se avançou ao investigar como o ambiente laboratorial dos jogos de empresas emulou a instituição do leilão pós-oferta, cujo sistema microeconômico foi devidamente identificado. Também foi apresentado como as preferências foram controladas nos jogos de empresas analisados. Identificou-se ainda que os prin-

QUADRO 5 - Controle de preferências nos jogos de empresas

\begin{tabular}{|c|c|c|}
\hline Princípio & Descrição do princípio & Incentivos \\
\hline Não saciedade & $\begin{array}{c}\text { Agentes são não saciáveis quanto ao retorno } \\
\text { médio da atividade }\end{array}$ & Crédito de disciplina vinculado a TIR \\
\hline Saliência & Agentes têm clara percepção de causa e efeito & $\begin{array}{l}\text { Testes objetivos de conhecimento, rodadas-teste, } \\
\text { análise anual de resultados }\end{array}$ \\
\hline Dominância & $\begin{array}{c}\text { Retorno médio do experimento é sempre maior } \\
\text { que outros estímulos }\end{array}$ & Decisões realizadas em sala de aula \\
\hline Privacidade & $\begin{array}{l}\text { Agentes têm acesso apenas as suas } \\
\text { alternativas de decisão e recompensa }\end{array}$ & $\begin{array}{c}\text { Proporcionado pelo simulador, com controle de acesso } \\
\text { individual }\end{array}$ \\
\hline
\end{tabular}

Fonte: Os autores do estudo. 
cipais elementos que propiciam o contexto organizacional nos jogos de empresas são a interação de múltiplas variáveis, o comportamento social e o envolvimento do participante com o caso empresarial.

No âmbito teórico da economia experimental, identifica-se que a principal contribuição desses estudos seja a formalização do ambiente laboratorial dos jogos de empresas, que pode ser equiparada aos tipos de experimentos econômicos propostos por Harrison e List (2004). Como se acredita que é possível conduzir estudos experimentais e não experimentais, optouse por denominar o ambiente laboratorial dos jogos de empresas como Ambiente Laboratorial Contextualizado (ALC). Acredita-se que tal ambiente, equiparado aos experimentos econômicos, seja um avanço aos experimentos de laboratório, conforme apresentado na Figura 3.

$\mathrm{O}$ que Edman (2005) aventou foi aqui aprofundado com indícios e evidências, ou seja, os jogos de empresas se aproximam mais dos dados que ocorrem em ambiente natural. Ressalva-se, contudo, que, diferentemente dos outros experimentos econômicos presentes nesta classificação, que não se restringem a uma instituição, o ALC é condicionado à instituição do leilão pós -oferta. Verifica-se ainda que o ALC pode avançar em termos de classificação, ao sofisticar o desenho da pesquisa, de modo que utilize profissionais em vez de estudantes, como sujeitos na pesquisa, permitindo que $\circ$ ambiente avance para $\circ$ ser superior ao experimento de campo artefactual (ECA). Pelo fato de o experimento de campo framed (ECF) utilizar o cenário real, ou seja, numa indústria de competição de mercado deveria utilizar produtos físicos para comercialização, não é possível que o ALC seja superior ao ECF. A utilização de profissionais como sujeito nos jogos de empresas é possível no âmbito da pós-graduação lato-sensu ou stricto sensu, conforme utilizado no pré-experimento do Estudo I e na Fase I do Estudo 3. Desse modo, os jogos de empresas conduzidos no âmbito da pós-graduação proporcionam maior validade externa, sendo superior ao ECA.

Vale ressaltar ainda a possibilidade da utilização do ALC para a realização de estudos não experimentais, como os conduzidos em estudos anteriores que utilizaram jogos de empresas. Para estudos não ex-

\section{Dados em ambiente controlado \\ Estudantes \\ Profissionais \\ Dados em ambiente natural

EL $\underline{\text { ALC }} \quad$ ECA $\underline{\underline{A L C}}$ ECF $\quad$ ECN

Legenda:

EL: Experimento de Laboratório

ALC: Ambiente Laboratorial Contextualizado

ECA: Experimento de Campo Artefactual

ECF: Experimento de Campo Eramed

ECN: Experimento de Campo Natural

FIGURA 3 - Contribuição dos jogos de empresas quanto aos ambientes laboratoriais

Fonte: Adaptado de List (2007). 
perimentais identificou-se como o principal benefício a possibilidade de análise com diversas variáveis, tal como o Estudo 2, que dispunha de 14 variáveis.

\section{CONSIDERAÇÕES FINAIS}

Conclui-se que a pesquisa realizada neste artigo obteve êxito em vista do seu objetivo geral. A investigação foi conduzida com o objetivo de verificar se jogos de empresas seriam ambientes laboratoriais adequados para a condução de pesquisas econômicas. Verificou-se que, pelos resultados dos estudos empíricos analisados, os jogos de empresas foram ambientes laboratoriais adequados quando se investigaram problemas que utilizaram a instituição do leilão pós-oferta, como analisado nos estudos I, 2 e 3.Também foi adequado em estudos que avaliaram a temática do oligopólio e poder de mercado, tal como evidenciado no Estudo 2.

Foi possível ainda concluir que a utilização do ambiente laboratorial dos jogos de empresas é adequada quando utilizar hipóteses elaboradas por estudos anteriores, em vista da limitação quanto à validade interna, e indicada para avaliar validade externa das hipóteses investigadas, principalmente para verificar o efeito do contexto organizacional produzido por três elementos que são a interação de múltiplas variáveis, o comportamento social espontâneo e induzido e o envolvimento pessoal racional-emocional propiciado pelo caso empresarial.

Como principal limitação das conclusões delineadas, cita-se o escopo dos estudos investigados, sendo necessária sua ampliação para reforçar os argumentos. Pode-se analisar o comportamento de variáveis advindas de modelos teóricos ou estudos anteriores sob o efeito desses elementos, ou ainda o efeito deles e sua interação. Esses elementos, em conjunto, permitem aumentar a validade externa de resultados obtidos no ambiente laboratorial dos jogos de empresas. Também indicam-se os jogos de empresas como ambientes laboratoriais para pesquisa, quando da realização de pesquisas não experimentais que sejam possíveis a partir de variáveis presentes nos demonstrativos emitidos pelo simulador. 


\section{REFERÊNCIAS}

BATTISTELLA, Luciana Flores; SCHUSTER, Marcelo da Silva; DIAS, Valéria da Veiga. Análise da evolução das publicações do tema justiça organizacional no Brasil: um estudo bibliométrico nos eventos da Anpad e do Semead. Estudos do ISCA, [S. I.], n. 4, 2012.

BRUNS, William J. Business Games for Management Research. In Academy of Management Proceedings, [S. I.], p. I48-155, 1962.

CAMERON, Kim S.; WHETTEN, David. A. Perceptions of organizational effectiveness over organizational life cycles. Administrative Science Quarterly, [S. I.], v. 26, n. 4, 525-544. 1981.

CHAMBERLIN, Edward. H. An experimental imperfect market. The Journal of Political Economy, [S. I.], v. 6I, n. 2, p. 95- I08. 1948.

CLAY, Karen B.;TROESKEN, Werner. Further tests of static oligopoly models: whiskey, I882-1898. Journal of Industrial Economics, [S. I.], v. 5I, n. 2, p. I5I-166, 2003.

CROSON, Rachel; GAECHTER, Simon. The science of experimental economics. Journal of Economic Behavior \& Organization, [S. I.], v. 73, n. I, p. |22-I3I, Jan. 2010.

SCHMITT, Camila S.;VON DER HAYDE, Cristian Tadeu; DREHER, Marialva Tomio. Sustentabilidade como vantagem competitiva: uma análise bibliométrica. Revista Eletrônica de Estratégia \& Negócios, [S. I.], v. 6, n. 2, p. p. I57-174, 2013.

DAVIS, Douglas; HOLT, Charles A. Experimental economics. Princeton: Princeton University Press, 1992.

DILL, William R.; DOPPELT, Neil. The acquisition of experience in a complex management game. Management Science, [S. I.], v. I0, n. I, 30-46, 1963.

EDMAN, Jan. Capabilities of Experimental Business Gaming. Developments in Business Simulation and Experiential Learning, [S. I.], v. 32, p. 104-109, 2005.

ENGEL, Christoph. How much collusion? A meta-analysis of oligopoly experiments. Journal of Competition Law and Economics, [S. I.], v. 3, n. 4, p. 49I-549, 2007.

FARIA, Anthony J. et al. Developments in Business Gaming A Review of the Past 40 Years. Simulation \& Gaming, [S. I.], v. 40, n. 4, p. $464-$ 487, 2009.

FERREIRA, Vera Rita Mello. Psicologia econômica: como o comportamento econômico influencia nas nossas decisões. Rio de Janeiro: Elsevier, 2008.

FRECHETTE, Guillaume R., Laboratory Experiments: Professionals Versus Students. October, 2011. Disponível em: SSRN:<http://ssrn.com/abstract $=|9392| 9\rangle$. Acesso em: 02 dez. 2014.

FRIEDMAN, Daniel ; SUNDER, Shyam. Experimental Methods: A primer for Economists. New York: Cambridge University Press, 1994.

GONÇALVES, Elisa Pereira. Iniciação à pesquisa científica. 4. ed. Campinas:Alínea, 2007.

GRECO, Marco; BALDISSIN, Nicola; NONINO, Fabio. An exploratory taxonomy of business games. Simulation \& Gaming, [S. I.], v. 44, n. 5, p. 645-682, 2013.

HALPIN, Annette L. A review of the simulation research in the Academy of Management Journal: Suggestions for strengthening the research conducted by ABSEL members. Developments in Business Simulation and Experiential Learning, [S. I.], v. 40, p. I54-165, 2013.

HARRISON, Glenn W.; LIST, John A. Field experiments. Journal of Economic Literature, [S. I.], v. 42, n. 4, p. 1009-1055, 2004.

HARRISON, Glenn W.; LIST, John A. Naturally Occurring Markets and Exogenous Laboratory Experiments: A Case Study of the Winner's Curse. The Economic Journal, [S. I.], v. II8, n. 528, p. 822-843, 2008.

HEUKELOM, Floris. How validity travelled to economic experimenting. Journal of Economic Methodology, [S. I.], v. I8, n. I, p. 13-28, 2011.

HOUAISS, Antônio. Dicionário eletrônico da língua portuguesa. Objetiva, 2007.

KEYS, Bernard; WOLFE, Joseph. The role of management games and simulations in education and research. Journal of management, [S. I.], v. 16, n. 2, p. 307-336, 1990.

LANE, David C. On a resurgence of management simulations and games. Journal of the Operational Research Society, [S. I.], v. 46, n. 5, p. 604-625. 1995.

LANT, Theresa K. Simulation games: A research method for studying organizational behavior. Unpublished manuscript. New York: New York University, 1989.

LIST, John A. Field experiments: a bridge between lab and naturally occurring data. The BE Journal of Economic Analysis \& Policy, [S. I.], v. 5, n. 2, 2007.

LIST, John A. et al. So You Want to Run an Experiment, Now what? Some Simple Rules of Thumb for Optimal Ex- 
perimental Design. Experimental Economics, [S. I.], v. I4, n. 4, p. 439-457, 201 I.

LOEWENSTEIN, George. Experimental economics from the vantage-point of behavioural economics. The Economic Journal, [S. I.], v. 109, n. 453, p. 25-34, 1999.

MICHALISIN, Michael D. et al. Top Management Team Cohesion and Superior Industry Returns An Empirical Study of the Resource -Based View. Group \& Organization Management, [S. I.], v. 29, n. I, p. I25- I40, 2004.

MOORE, Don A.; SWIFT, Samuel A. The three faces of overconfidence in organizations. Social Psychology and Organizations, [S. I.], p. |47-184, 2010.

MOORE, Don A.; HEALY, Paul J. The trouble with overconfidence. Psychological Review, [S. I.], v. II5, n. 2, p. 502-5 I 7, 2008.

ORTMANN,Andreas; GIGERENZER,

Gerd. Reasoning in economics and psychology: Why social context matters. In: STREIT, Manfred E. ; MUMMERT, Uwe ; KIWIT, Daniel (Ed.). Cognition, Rationality, and Institutions. Berlin: Springer, 1997. p. I31-145.

PEÇANHA, Reynaldo Schirmer; IIZUKA, Edson Sadao. Análise da produção científica brasileira sobre sustentabilidade entre os anos de 2008 a 20 I I. Revista de Gestão Ambiental e Sustentabilidade-GeAS, [S. I.], v. 3, n. I, p. $01-17,20 \mid 4$.

PLOTT, Charles R.; SMITH, Vernon (Ed.). Handbook of experimental economics results. [S. I.]: North-Holland, 2008.
RIBEIRO, Henrique César Melo; COSTA, Benny Kramer; FERREIRA, Manuel Portugal. Produção acadêmica dos temas estratégia e governança corporativa. Revista de Administração FACES Journal, Belo Horizonte, v. I3, n. 3, p. 28-46, jul./set. 2014.

SAUAIA, A. C. A. Laboratório de Gestão: simulador organizacional, jogo de empresas e pesquisa aplicada. 3. ed. Manole: Barueri, 2013.

SAUAIA, A. C. A. Regras institucionais e desempenho setorial: um estudo laboratorial com jogos de empresas. In: ENCONTRO DA ASSOCIAÇÃO NACIONAL DE PÓS-GRADUAÇÃO E PESQUISA EM ADMINISTRAÇÃO, 35. 20I I, Rio de Janeiro. Anais... Rio de Janeiro:ANPAD, 20I I.

SILVA, A. M. Estrutura de mercado e comportamento gerencial na convergência de preços: um estudo com jogos de empresas. In: SEMEAD - SEMINÁRIOS EM ADMINISTRAÇÃO FEA/USP, I7., 20|4, São Paulo. Anais... São Paulo: SEMEAD, 2014.

SILVA, A. M.; SAUAIA, A. C. Aferição do poder de mercado: um estudo experimental com os modelos ECD e NOIE. Revista de Economia e Administração, [S. I.], v. I2, n. 4, p. 456-479, 2013.

SILVA, Adriano Maniçoba; SAUAIA, Antonio Carlos Aidar. Desempenho passado e nível de confiança de gestores: um estudo laboratorial. In: SEMEAD - SEMINÁRIOS EM ADMINISTRAÇÃO FEA/USP, 15., 2012, São Paulo. Anais... São Paulo: SEMEAD, 2012.
SILVA, Paula Maines. Abordagem multinível em redes: análise da produção cientifica. Revista de Administração FACES Journal, Belo Horizonte, v. I4, n. I, p. |43-I5I, jan./mar. 2015.

SIMCO 6.0 - Simulação Comercial. Manual da Empresa. Bernard Sistemas Ltda. Florianópolis: [s. n.], 2009.

SMITH, V. An empirical study of decentralized institutions of monopoly restraint. Essays in Contemporary Fields of Economics: In Honor of Emanuel T. Weiler (1914-1979). Purdue West Lafayette: University Press, 198I. p. 83- 106.

SMITH, V. Microeconomic systems as an experimental science. The American Economic Review, [S. I.], v. 72, n. 5, p. 923-955, 1982.

SMITH, Vernon. L. An experimental study of competitive market behavior. The Journal of Political Economy, [S. I.], v. 70, n. 2, p. I I I-137, 1962.

STAINTON, Andrew J.; JOHNSON, Johnnie E.; BORODZICZ, Edward P. Educational validity of business gaming simulation: A research methodology framework. Simulation \& Gaming, [S. I.], v. 4I, n. 5, p. 705-723, 2010.

WOLFE, Joseph; CASTROGIOVANNI, Gary. Business games as strategic management laboratories. Developments in Business Simulation and Experiential Learning, [S. I.], v. 33, p. 3I-40, 2006. ZIZZO, Daniel John. Experimenter demand effects in economic experiments. Experimental Economics, [S. I.], v. I3, n. I, p. 75-98, 2010. 